\title{
Optimal photon energies for initialization of hybrid spin quantum registers of NV centers in diamond
}

\author{
K. Rama Koteswara Rao ${ }^{1,2}$, Yihua Wang ${ }^{1}$, Jingfu Zhang ${ }^{1}$, and Dieter Suter ${ }^{1}$ \\ ${ }^{1}$ Fakultät Physik, Technische Universität Dortmund, D-44221 Dortmund, Germany \\ ${ }^{2}$ Department of Physics, Bennett University, Greater Noida 201310, India
}

(Dated: April 6, 2020)

\begin{abstract}
Initializing quantum registers with high fidelity is a fundamental precondition for many applications like quantum information processing and sensing. The electronic and nuclear spins of a Nitrogen-Vacancy (NV) center in diamond form an interesting hybrid quantum register that can be initialized by a combination of laser, microwave, and radio-frequency pulses. However, the laser illumination, which is necessary for achieving electron spin polarization, also has the unwanted sideeffect of depolarizing the nuclear spin. Here, we study how the depolarization dynamics of the ${ }^{14} \mathrm{~N}$ nuclear spin depends on the laser wavelength. We show experimentally that excitation with an orange laser $(594 \mathrm{~nm})$ causes significantly less nuclear spin depolarization compared to the green laser $(532 \mathrm{~nm})$ typically used for excitation and hence leads to higher nuclear spin polarization. This could be because orange light excitation inhibits ionization of $\mathrm{NV}^{0}$ into $\mathrm{NV}^{-}$and therefore suppresses one source of noise acting on the nuclear spin.

PACS numbers: 03.67.Lx, 76.70.Hb, 33.35.+r, 61.72.J-
\end{abstract}

\section{INTRODUCTION}

Nitrogen-Vacancy (NV) centers in diamond have interesting properties for spin based quantum information processing and nano-scale Nuclear Magnetic Resonance (NMR) spectroscopy and imaging [1 8]. Nuclear spins coupled to NV centers are useful resources for these applications. They can be used as qubits in a hybrid quantum register [2, 4, 9, 11] or as long-lived memories to store quantum states of electron spins [12 14. They can also be used as a channel for transferring polarization between electron spins of NV centers and nuclear spins of the bulk (remotely coupled) in hyperpolarization experiments [15] 18. Initializing or polarizing nuclear spins is an essential part of these experiments.

Electron spins of NV centers can be polarized near completely by optical pumping. However, this process does not automatically lead to the polarization of nuclear spins coupled to NV centers. Different methods for polarizing these nuclear spins have been discussed in the literature [14, 19 23. One of them makes use of a level anti-crossing in the excited state which occurs in a magnetic field of $51.2 \mathrm{mT}$ oriented along the $\mathrm{NV}$ axis [19. Anti-crossing at this magnetic field causes mixing between the electron and nuclear spin states of the excited state and leads to polarization of both the electron and nuclear spins under optical pumping. This method was successfully used to polarize the ${ }^{14} \mathrm{~N}$ nuclear spin of an NV center and a ${ }^{13} \mathrm{C}$ nuclear spin of the first coordination shell. However, this method does not lead to good polarization of other ${ }^{13} \mathrm{C}$ nuclear spins [24, and it is only applicable at one specific strength and orientation of the magnetic field. Another interesting method to initialize nuclear spins of NV centers is through single shot readout [20]. This method has been used to initialize the ${ }^{14} \mathrm{~N}$ nuclear spin of an NV center and also a specific ${ }^{13} \mathrm{C}$ nuclear spin [25. However, this method also requires a strong static magnetic field compared to the transverse components of the hyperfine interaction [20].

A more general method to polarize nuclear spins coupled to NV centers is to apply a sequence of microwave (MW), radio-frequency (RF), and laser pulses [14, 21 23. The basic idea of this method is to first polarize the electron spin and then transfer this polarization to a nuclear spin coupled to it, using MW and RF pulses. The electron spin, which is then left in a mixed state, can be repolarized by a second laser pulse. However, this laser pulse causes depolarization of the nuclear spin and the degree of depolarization depends on the power and duration of the laser pulse. One possible source of nuclear spin depolarization is the ionization of the NV center during the laser pulse.

In this work, we study the depolarization dynamics of the ${ }^{14} \mathrm{~N}$ nuclear spin of an NV center for different wavelengths of laser illumination including 532 and $594 \mathrm{~nm}$. The absorption cross-section of the NV center at both wavelengths is roughly the same, but they cause very different ionization rates: The $532 \mathrm{~nm}$ photons can ionize $\mathrm{NV}^{0}$ into $\mathrm{NV}^{-}$and vice-versa by a two-photon process, while the $594 \mathrm{~nm}$ photons can ionize $\mathrm{NV}^{-}$into $\mathrm{NV}^{0}$ but the probability rate from $\mathrm{NV}^{0}$ into $\mathrm{NV}^{-}$is very small at this wavelength [26. Here, we show experimentally that the depolarization rate of the ${ }^{14} \mathrm{~N}$ nuclear spin is significantly lower for $594 \mathrm{~nm}$ irradiation than for $532 \mathrm{~nm}$, while the polarizing rate of the electron spin is roughly the same for both wavelengths, resulting in higher nuclear spin polarization under $594 \mathrm{~nm}$ excitation. Although all the wavelengths between 575 and $637 \mathrm{~nm}$ significantly inhibit ionization of $\mathrm{NV}^{0}$ into $\mathrm{NV}^{-}$, wavelengths around $594 \mathrm{~nm}$ are optimal for the present purpose because of the high absorption cross-section of $\mathrm{NV}^{-}$charge state at these wavelengths [26].

This paper is arranged as follows. In Section II, we describe the method for polarizing the nuclear spin and the 
differences between the green and orange light excitation of an NV center. In Section III, we give the details of our experiment and discuss the results and in Section IV, we conclude.

\section{POLARIZATION METHOD}

We consider polarizing the single ${ }^{14} \mathrm{~N}(I=1)$ nuclear spin coupled to the electronic spin $S=1$. The Hamiltonian of such a system interacting with a static magnetic field aligned along the NV axis can be written as

$$
\begin{aligned}
\mathcal{H}= & D S_{z}^{2}+\gamma_{e} B S_{z}+\gamma_{n} B I_{z}+P I_{z}^{2} \\
& +A_{\|} S_{z} I_{z}+A_{\perp}\left(S_{x} I_{x}+S_{y} I_{y}\right) .
\end{aligned}
$$

Here, $S_{\alpha}$ and $I_{\alpha}$ represent the $\alpha$-components of the spin angular momenta of the electronic and nuclear spins respectively, and $\gamma_{e}$ and $\gamma_{n}$ are their respective gyromagnetic ratios. $D=2870 \mathrm{MHz}$ and $P=-4.95 \mathrm{MHz}$ 27. are the zero-field splitting of the electron spin and the quadrupole splitting of the ${ }^{14} \mathrm{~N}$ nucleus, measured in frequency units. $B$ represents the strength of the static magnetic field, and $A_{\|}=-2.3 \mathrm{MHz}$ and $A_{\perp}=-2.6 \mathrm{MHz}$ [28 30] are the components of the hyperfine interaction along the NV axis and perpendicular to it.

Fig. 1 shows a schematic representation of the method for polarizing the two spins. Fig. 11(a) shows the pulse sequence. The first laser pulse polarizes the electron spin into the $m_{s}=0$ state, but this leaves the nuclear spin in a mixed state as illustrated in Fig. 1(b). The polarization of the electron spin can be transferred to the nuclear spin by applying two electron spin transition selective MW $\pi$ pulses followed by another two nuclear spin transition selective RF $\pi$ pulses. Now, the nuclear spin is fully polarized, but the electron spin is in the completely mixed state. To repolarize the electron spin, we need to apply another laser pulse. However, this laser pulse causes partial depolarization of the nuclear spin [22]. The amount of depolarization depends on the intensity and duration of the laser pulse. There may be different sources of noise that cause depolarization of the nuclear spin. One possible source is the ionization of $\mathrm{NV}^{-}$into $\mathrm{NV}^{0}$ and viceversa during optical illumination: the electronic spin of $\mathrm{NV}^{0}$ is $S=1 / 2$ and its hyperfine interaction is different from that of $\mathrm{NV}^{-}$.

All the experiments that are reported so far use green light $(532$ or $520 \mathrm{~nm})$ to initialize and repolarize the NV center. It is known that under green light illumination, the charge state of an NV center flips between the $\mathrm{NV}^{-}$ and $\mathrm{NV}^{0}$ states with an average distribution of the $\mathrm{NV}^{-}$ and $\mathrm{NV}^{0}$ populations being 70 and $30 \%$ respectively [26]. This implies that by the end of the initialization laser pulse, the center would be in the $\mathrm{NV}^{0}$ state with $30 \%$ probability. In this case, the subsequent $\mathrm{MW}$ and $\mathrm{RF}$ pulses have no effect on the spin. However, the repolarizing laser pulse can convert it into $\mathrm{NV}^{-}$and this state

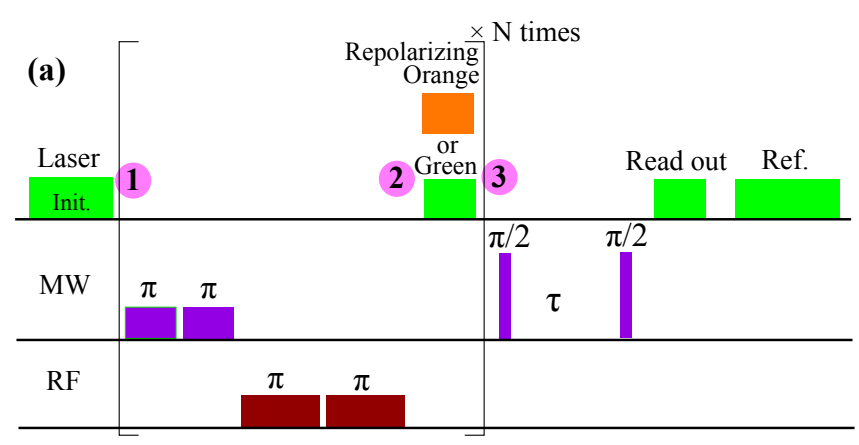

(b)
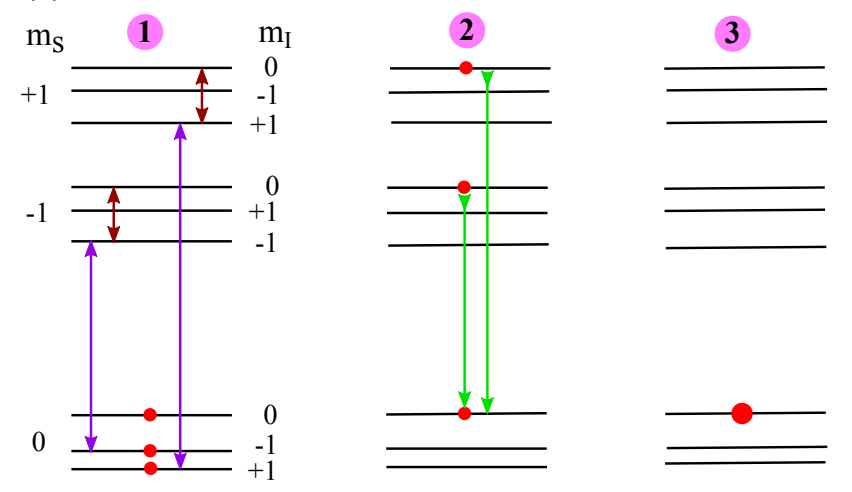

FIG. 1. Schematic representation of the polarization method. (a) Pulse sequence; green or orange rectangle in the first line represent corresponding laser pulses. The rectangles in the second and third row represent $\mathrm{MW}$ and RF pulses applied at resonance to the electronic and nuclear spin transitions respectively. The $\pi / 2-\tau-\pi / 2$ sequence in the second line is used to measure the free-induction decay of the electron spin. (b) Energy level diagram and corresponding populations at different stages of the pulse sequence

contributes to the observed signal. Since polarization transfer does not occur for this, signal contribution from it results in reduced polarization of the nuclear spin.

In order to eliminate this depolarization channel, we therefore change the protocol: for the repolarization laser pulse, we use an orange laser, operating at $594 \mathrm{~nm}$, instead of the conventional green laser. As we show in the following, this leads to a significant reduction of the depolarization process and results in higher nuclear spin polarization. The absorption cross-section of $\mathrm{NV}^{-}$is roughly the same for both lasers, but the orange light does not result in ionization of $\mathrm{NV}^{0}$ into $\mathrm{NV}^{-}[26]$. Accordingly, it avoids signal contribution if the center's charge state is changed during the repolarizing pulse. An important point to note here is that a single NV center, under green light readout, generates very little fluorescence attributable to its $\mathrm{NV}^{0}$ state [26].

Fig. 2 illustrates the relative positions of the energy levels of $\mathrm{NV}^{-}$and $\mathrm{NV}^{0}$ in the band gap of diamond. The Zero-Phonon Lines (ZPL) of the $\mathrm{NV}^{-}$and $\mathrm{NV}^{0}$ charge states occur at 637 and $575 \mathrm{~nm}$, respectively. This implies that photons of wavelength 532 or $520 \mathrm{~nm}$ can excite 


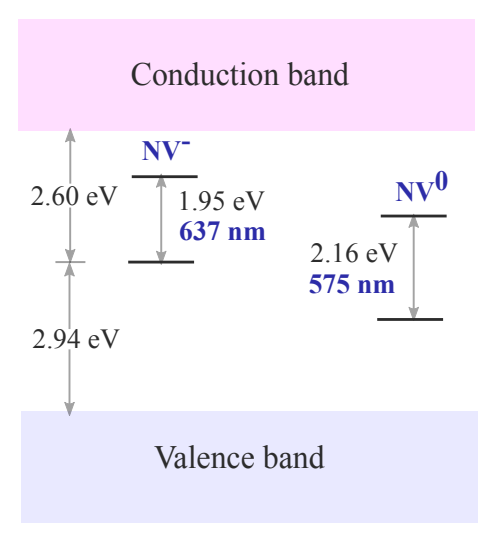

FIG. 2. Schematic representation of the energy levels of an $\mathrm{NV}$ center for its negative and neutral charge states in the band gap of diamond.

both charge states and also can ionize one into the other. However, photons of wavelength $594 \mathrm{~nm}$ can excite the $\mathrm{NV}^{-}$state, but not $\mathrm{NV}^{0}$. Since photo-induced ionization of an NV center at the mentioned wavelengths by a two-photon process necessitates its excitation from the ground to the excited state, $594 \mathrm{~nm}$ light can only ionize $\mathrm{NV}^{-}$into $\mathrm{NV}^{0}$, but not the other way [26]. This is true for all wavelengths between 575 and $637 \mathrm{~nm}$. Since the $\mathrm{NV}^{-}$charge state has a high absorption cross-section around $590 \mathrm{~nm}$, allowing fast polarization of its electron spin [26], excitation with a wavelength around $590 \mathrm{~nm}$ should be optimal for the present purpose.

\section{EXPERIMENTAL RESULTS}

All experiments have been performed on a single NV center from a $99.99 \%{ }^{12} \mathrm{C}$ enriched bulk diamond sample with a nitrogen concentration of $<5 \mathrm{ppb}$. These experiments were also repeated on another center from the same sample and the results are very similar. The setup used for these experiments was based on a homebuilt optical confocal microscope equipped with 520, 532 and $594 \mathrm{~nm}$ lasers for optical excitation of the NV center and MW and RF electronics for resonant excitation of electron and nuclear spins. The fluorescence of the NV center was collected through a $605 \mathrm{~nm}$ dichroic mirror followed by a $594 \mathrm{~nm}$ long pass filter. An electromagnet was used to apply a static magnetic field of $2.8 \mathrm{mT}$ oriented along the NV axis.

The pulse sequence given in Fig. 1 was implemented in the following way. A $4 \mu$ s long 520 or $532 \mathrm{~nm}$ laser pulse was applied to initialize the charge and spin states of the NV center into the $\mathrm{NV}^{-}, m_{s}=0$ states. The following MW $\pi$ pulses were applied to the transitions $\left|m_{s}, m_{I}\right\rangle=|0,-1\rangle \longleftrightarrow|-1,-1\rangle$, and $|0,+1\rangle \longleftrightarrow$ $|+1,+1\rangle$, whose frequencies were 2789.13 and 2947.42 $\mathrm{MHz}$ respectively. The duration of each of these pulses was $1 \mu \mathrm{s}$. The RF $\pi$ pulses were applied to the nu-

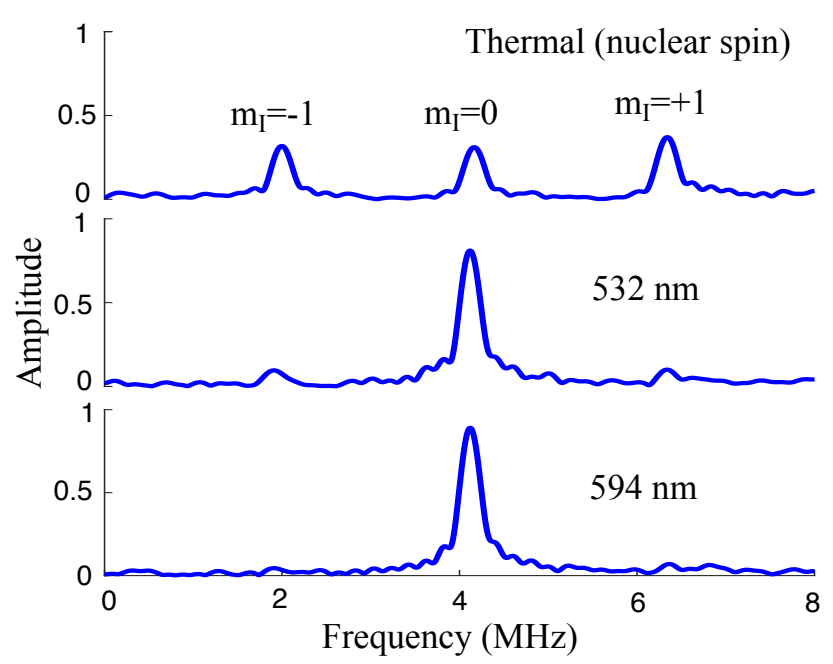

FIG. 3. Fourier transforms of electron spin FIDs measured between the $m_{s}=0$ and -1 subspaces. The top row corresponds to the spectrum with thermal nuclear spin polarization, the middle and bottom rows correspond to the spectra obtained after the nuclear spin polarizing pulse sequence of Fig. 1 with $N=4$ (cycles) for $532 \mathrm{~nm}$ (duration, $500 \mathrm{~ns}$ ) and $594 \mathrm{~nm}$ (700 ns) illumination respectively.

clear spin transitions, $|-1,-1\rangle \longleftrightarrow|-1,0\rangle,|+1,+1\rangle \longleftrightarrow$ $|+1,0\rangle$, whose frequencies were 7.1064 and $7.1226 \mathrm{MHz}$ respectively, and the duration of each of these pulses was $62 \mu \mathrm{s}$. The repolarizing laser pulse was derived from the 520,532 or $594 \mathrm{~nm}$ laser. Then, an electron spin free-induction decay (FID) was measured by applying the Ramsey sequence $(\pi / 2-\tau-\pi / 2)$ between the $m_{s}=0$ and -1 subspaces followed by a 400 ns readout laser pulse. Here, the $\pi / 2 \mathrm{MW}$ pulses were non-selective and excited all allowed transitions between these subspaces. Since the repolarizing laser pulse brings the populations of the $m_{s}=-1$ and +1 subspaces into the $m_{s}=0$ subspace, the intensities of the spectral lines obtained by Fourier transforming the free-induction decay represent populations of the corresponding nuclear spin sub-levels. For the experiments involving 532 and $594 \mathrm{~nm}$ repolarizing laser pulses, the initialization and readout pulses were derived from the $532 \mathrm{~nm}$ laser and for those involving $520 \mathrm{~nm}$ repolarizing laser pulse, the same $520 \mathrm{~nm}$ laser was used for initialization and readout.

The spectra obtained by applying the pulse sequence of Fig. 1 with $N=4$ cycles of polarization transfer and repolarizing pulses for 532 and $594 \mathrm{~nm}$ repolarizing illumination are shown in Fig. 3, together with a spectrum showing thermal nuclear spin polarization. This spectrum was obtained by applying the initializing laser pulse followed directly by the Ramsey sequence (i.e. $N=0$ ). It contains three lines corresponding to the three ${ }^{14} \mathrm{~N}$ nuclear spin states, $m_{I}=-1,0$, and +1 , which are split by the hyperfine coupling. These three lines have roughly equal amplitude which implies that the nuclear spin is in the maximally mixed state after the initializing 

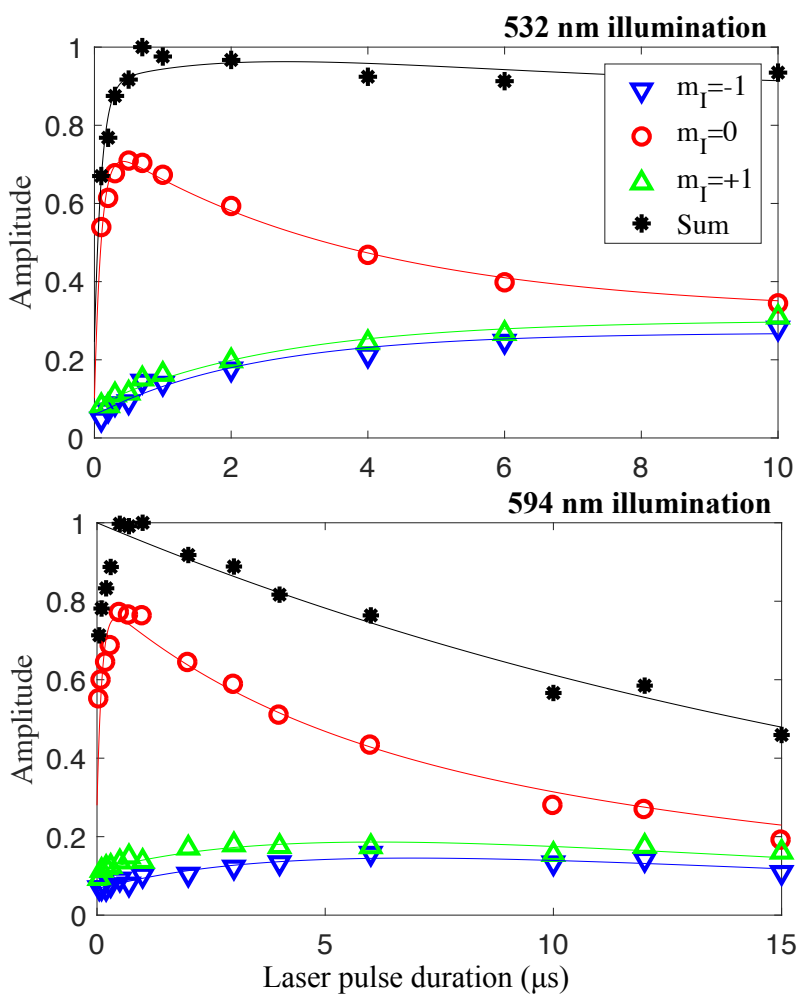

FIG. 4. Dynamics of electron spin polarization and nuclear spin depolarization as a function of the duration of the repolarizing laser pulse. Circles, lower, and upper triangles represent experimental data corresponding to the populations of the $m_{I}=0,-1$, and +1 states of the ${ }^{14} \mathrm{~N}$ nuclear spin respectively, and asterisks represent the total population. The experimental data are compared to the fit with the model given in Ref. 22. (Appendix) and the corresponding time constants are given in Table I.

pulse. The spectrum corresponding to the $532 \mathrm{~nm}$ repolarizing illumination shows significantly decreased outer peaks and an increased central peak, which implies that the population of $m_{I}=-1$ and $m_{I}=+1$ states is transferred to the $m_{I}=0$ state. The spectrum corresponding to the $594 \mathrm{~nm}$ repolarizing illumination shows almost no outer peaks and a strong central peak. We calculate the nuclear spin polarization $(p)$ by writing its density matrix as $p|0\rangle\langle 0|+(1-p) I$, where $I$ is the $3 \times 3$ identity matrix. From the spectra, we obtain $p$ as $76.3( \pm 1.9) \%$ and $89.0( \pm 2.7) \%$ for the 532 and $594 \mathrm{~nm}$ repolarizing illumination respectively.

The amplitudes of the three spectral lines and their sum as a function of the repolarizing laser pulse duration are shown in Fig. 4 for a single cycle of polarization transfer and repolarization. The amplitudes of all three lines increase initially, indicating that the electron spin polarization increases. The central line $\left(m_{I}=0\right)$ reaches its maximum after $\approx 500$ ns and then starts to decrease, whereas the outer lines $\left(m_{I}=-1\right.$ and +1$)$ continue to grow. This indicates that the polarization of the nuclear spin decreases. The sum of the amplitudes, after reaching

\begin{tabular}{|c|c|c|c|}
\hline $\begin{array}{c}\text { Wavelength } \\
(\mathrm{nm})\end{array}$ & $\begin{array}{c}\text { Electron spin } \\
\text { polarization } \\
\text { time constant } \\
(\mathrm{ns})\end{array}$ & $\begin{array}{c}\text { Nuclear spin } \\
\text { depolarization } \\
\text { time constant } \\
(\mu \mathrm{s})\end{array}$ & $\begin{array}{c}\text { Decay time of } \\
\mathrm{NV}^{-} \\
\text {population } \\
(\mu \mathrm{s})\end{array}$ \\
\hline \hline 520 & $170( \pm 21)$ & $6.4( \pm 1.1)$ & $\mathrm{NA}$ \\
\hline 532 & $101( \pm 16)$ & $8.4( \pm 2.5)$ & $\mathrm{NA}$ \\
\hline 594 & $110( \pm 22)$ & $16.6( \pm 4.8)$ & $20.4( \pm 1.0)$ \\
\hline
\end{tabular}

TABLE I. Electron spin polarization and ${ }^{14} \mathrm{~N}$ nuclear spin depolarization time constants for different wavelengths. The time constant corresponding to the decay of the $\mathrm{NV}^{-}$population for the $594 \mathrm{~nm}$ illumination is also given. The laser powers for $520 \mathrm{~nm}$ and $532 \mathrm{~nm}$ are $\approx 110$ and $90 \mu \mathrm{W}$ respectively. They are chosen such that the fluorescence count rate is half of the saturation value. The laser power for $594 \mathrm{~nm}$ is $\approx 80 \mu \mathrm{W}$.

its maximum value around $500 \mathrm{~ns}$, stays roughly constant for the $532 \mathrm{~nm}$ illumination, whereas for the $594 \mathrm{~nm}$ illumination it starts to decrease. This decay can be fit to an exponentially decaying function with a time constant of $20.4 \mu \mathrm{s}$. This decay reflects a decrease of the $\mathrm{NV}^{-}$population. Its time constant is more than an order of magnitude longer than the time needed to repolarize the center and hence does not cause significant loss of signal. The rate constants for the polarization and depolarization can be obtained by fitting the data to the model given in Ref. 22 (Appendix). The time constants for the polarization of the electron spin and the depolarization of the ${ }^{14} \mathrm{~N} n u-$ clear spin for different wavelengths are given in Table I. The polarization rates for the wavelengths 532 and 594 $\mathrm{nm}$ are very similar but faster compared to the one with $520 \mathrm{~nm}$ illumination. However the depolarization rate is significantly slower for the $594 \mathrm{~nm}$ illumination compared to the 520 and $532 \mathrm{~nm}$ ones. This implies that one should be able to reach higher nuclear spin polarization with $594 \mathrm{~nm}$ light and it explains the results of Fig. 3 .

The nuclear spin polarization measured from the data of Fig. 4 is shown in Fig. 5 as a function of the laser pulse duration. It clearly shows that the nuclear spin depolarization is slower for $594 \mathrm{~nm}$ illumination than for $532 \mathrm{~nm}$.

Even higher polarizations can be achieved by iterating the transfer-repolarization cycle, as indicated in Fig. 1. Fig. 6 shows the nuclear spin polarization as a function of the number of cycles for both the 532 and 594 $\mathrm{nm}$ repolarizing illuminations. The polarization increases with the number of cycles for both the cases and reaches its maximum value after 4 (3) cycles for the 594 (532) $\mathrm{nm}$ illumination. The low value of the nuclear spin polarization for $\mathrm{N}=1$ could be attributed to the imperfect MW and RF pulses and other experimental non-idealities which leave significant population in the $m_{I}=-1$ and +1 states. By iterating the transfer-repolarization cycle this population can be repumped into the $m_{I}=0$ state and hence improves the nuclear spin polarization.

Orange light illumination improves the ${ }^{14} \mathrm{~N}$ nuclear spin polarization but it also leads to loss of total sig- 


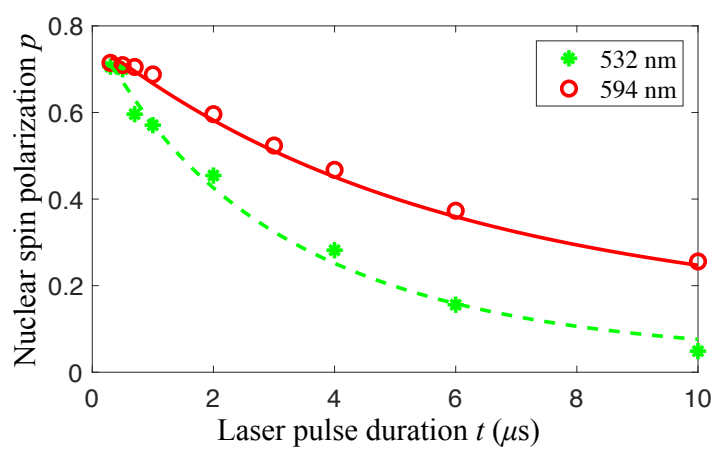

FIG. 5. Nuclear spin polarization as a function of laser pulse duration for 532 and $594 \mathrm{~nm}$ illumination. Circles and asterisks represent the experimental data, solid and dashed curves are the corresponding fits according to the model given in Appendix.

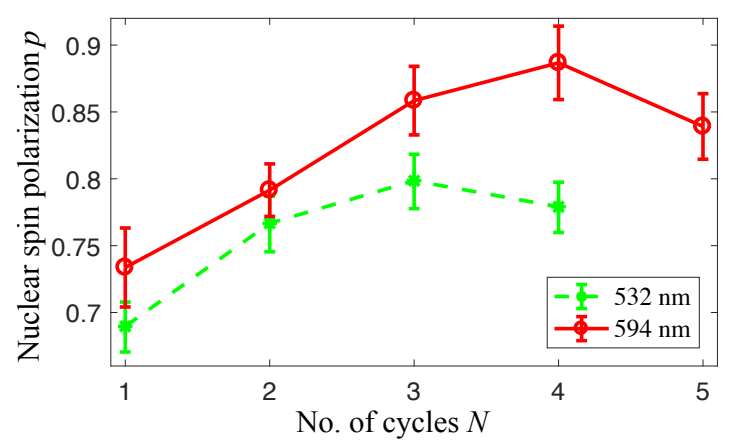

FIG. 6. Nuclear spin polarization versus $N$, the number of polarization transfer and repolarization cycles. The duration of the repolarizing laser pulse is 700 (500) ns for 594 (532) nm illumination.

nal. Here, we analyze the data given in Fig. 3 to understand signal loss and gain in polarization. From this data, the signal of the electron spin, in the order of nuclear spin states $m_{I}=-1,0,+1$, can be written as [9.96 94.61 8.49] (for green repolarizing pulses) and [3.38 95.73 4.61] (for orange repolarizing pulses). These signals are proportional to the populations of the corresponding nuclear spin states. So, the total signal loss is $\sim 8 \%$ and the gain in polarization is $\sim 13 \%$. However, the signal of the $m_{I}=0$ state is roughly equal in both cases and the loss of signal is only reflected in the $m_{I}=-1$ and +1 states. This implies, the signal that is lost is originally noise in the present scenario (of initializing quantum registers). In any case, the loss of signal can be compensated by increasing the averaging time by $17 \%$.

\section{DISCUSSION AND CONCLUSION}

Initializing single nuclear spins coupled to electron spins in solid state materials is an important prerequi- site for spin based hybrid quantum information processing and other applications like sensing. The approach described here, using a combination of laser (green, 532 or $520 \mathrm{~nm}$ ), MW, and RF pulses allows one to initialize nuclear spins coupled to NV centers in diamond at arbitrary magnetic fields. By judicious use of 532 and 594 $\mathrm{nm}$ (orange) laser pulses, we can achieve higher nuclear spin polarization $(89.0 \%$ ) than by using only $532 \mathrm{~nm}$ laser pulses $(76.3 \%)$. This polarization can be improved further by improving the fidelities of the transition selective MW and RF pulses. We have also studied the dynamics of electron spin polarization and nuclear spin depolarization under different wavelengths of laser illumination. We found that the electron spin polarization rates are similar for the laser wavelengths 532 and $594 \mathrm{~nm}$ and the nuclear spin depolarization rate under $594 \mathrm{~nm}$ illumination is significantly smaller than with $532 \mathrm{~nm}$. We believe the reason for different nuclear spin depolarization rates is related to the photo-induced ionization of the NV center. It is known that green light can ionize $\mathrm{NV}^{-}$into $\mathrm{NV}^{0}$ and vice-versa, and orange light also can ionize $\mathrm{NV}^{-}$into $\mathrm{NV}^{0}$, but the reverse processis less probable [26]. This combined with the previous observation [26, that a single $\mathrm{NV}$ center in the $\mathrm{NV}^{0}$ state yields very little fluorescence under green light irradiation can explain the observed slower nuclear spin depolarization under $594 \mathrm{~nm}$ illumination.

The method of polarizing nuclear spins by using a combination of MW, RF, and laser pulses has been previously applied to polarize ${ }^{13} \mathrm{C}$ nuclear spins as well [14, 23]. Here, we note that the achievable polarization by this method might also depend on the form of the hyperfine interaction. For the ${ }^{14} \mathrm{~N}$ nuclear spin, the hyperfine tensor contains no off diagonal elements [28 30] and the effect of the transverse (diagonal) components of the hyperfine tensor, which are off-diagonal to the Hamiltonian, can be neglected to an approximation. This implies that the nuclear spin eigenstates are approximately the Zeeman states. However, for ${ }^{13} \mathrm{C}$ nuclear spins, the hyperfine tensor in general contains off-diagonal elements, in particular terms that commute with the $z$-component of the electron spin angular momentum but not with the $z$-component of the nuclear spin angular momentum [24, 31, 32. This leads to mixing of nuclear spin Zeeman states within the $m_{S}=-1$ and +1 subspaces and for the $m_{S}=0$ subspace, Zeeman states are still approximately the eigenstates if the magnetic field is aligned with the $\mathrm{NV}$ axis. In any case, the use of green and orange light illumination should lead to higher nuclear spin polarization compared to green light illumination alone even for ${ }^{13} \mathrm{C}$ nuclear spins.

One disadvantage of the $594 \mathrm{~nm}$ excitation is the loss of $\mathrm{NV}^{-}$population, which corresponds to a reduction of the total signal. The loss of signal can be compensated by taking more averages, but the purity of a quantum state cannot be improved in a similar way. High purity quantum states are important for many applications. For example, the amount of entanglement that can be gen- 
erated in a system depends ultimately on the purity of its initial state 33 . High purity quantum states are also desirable for experiments involving fundamental tests of quantum mechanics such as temporal Bell inequality [34].

\section{ACKNOWLEDGMENTS}

This work was supported by the DFG through Grant No. Su 192/28-1. This project has received funding from the European Union's Horizon 2020 research and innovation programme under grant agreement No 828946. The publication reflects the opinion of the authors; the agency and the commission may not be held responsible for the information contained in it.

\section{APPENDIX}

\section{Rate equation model}

The rate equation model for the population dynamics of an NV center under laser illumination can be written as

$$
\frac{d}{d t} \vec{P}=\left(M\left(k_{S}, k_{I}\right)-k_{p}\right) \vec{P},
$$

where $k_{S}, k_{I}$, and $k_{p}$ represent the rate constants for the electron spin polarization, nuclear spin depolarization, and the decay of the $\mathrm{NV}^{-}$population respectively. $\vec{P}$ and $M\left(k_{S}, k_{I}\right)$ are the population vector and transition matrix respectively, defined as

$$
\begin{aligned}
\vec{P}= & \left(P_{\left|m_{S}, m_{I}\right\rangle=|0,+1\rangle}, P_{|0,-1\rangle}, P_{|0,0\rangle}, P_{|-1,-1\rangle},\right. \\
& \left.P_{|-1,+1\rangle}, P_{|-1,0\rangle}, P_{|+1,+1\rangle}, P_{|+1,-1\rangle}, P_{|+1,0\rangle}\right)
\end{aligned}
$$

$$
\begin{gathered}
M\left(k_{S}, k_{I}\right)= \\
{\left[\begin{array}{ccccccccc}
-2 k_{I} & k_{I} & k_{I} & 0 & k_{S} & 0 & k_{S} & 0 & 0 \\
k_{I} & -2 k_{I} & k_{I} & k_{S} & 0 & 0 & 0 & k_{S} & 0 \\
k_{I} & k_{I} & -2 k_{I} & 0 & 0 & k_{S} & 0 & 0 & k_{S} \\
0 & 0 & 0 & -k_{S} & 0 & 0 & 0 & 0 & 0 \\
0 & 0 & 0 & 0 & -k_{S} & 0 & 0 & 0 & 0 \\
0 & 0 & 0 & 0 & 0 & -k_{S} & 0 & 0 & 0 \\
0 & 0 & 0 & 0 & 0 & & -k_{S} & 0 & 0 \\
0 & 0 & 0 & 0 & 0 & 0 & 0 & -k_{S} & 0 \\
0 & 0 & 0 & 0 & 0 & 0 & 0 & 0 & -k_{S}
\end{array}\right] .}
\end{gathered}
$$

The population vector just before the repolarizing laser pulse can be written as $\vec{P}=\frac{1}{3}(0,0,1,0,0,1,0,0,1)$. Using this vector as the initial state, the solution to the rate equation model can be obtained as

$$
\begin{aligned}
& \vec{P}= \frac{1}{3} e^{-k_{p} t}\left(1-\frac{2 k_{I}}{\left(3 k_{I}-k_{S}\right)} e^{-k_{S} t}-\frac{\left(k_{I}-k_{S}\right)}{\left(3 k_{I}-k_{S}\right)} e^{-3 k_{I} t},\right. \\
& 1-\frac{2 k_{I}}{\left(3 k_{I}-k_{S}\right)} e^{-k_{S} t}-\frac{\left(k_{I}-k_{S}\right)}{\left(3 k_{I}-k_{S}\right)} e^{-3 k_{I} t} \\
& 1-\frac{2\left(k_{I}-k_{S}\right)}{\left(3 k_{I}-k_{S}\right)} e^{-k_{S} t}+\frac{2\left(k_{I}-k_{S}\right)}{\left(3 k_{I}-k_{S}\right)} e^{-3 k_{I} t} \\
&\left.0,0, e^{-k_{S} t}, 0,0, e^{-k_{S} t}\right) .
\end{aligned}
$$

This vector represents the population dynamics under the repolarizing laser pulse.
[1] L. Childress, M. V. Gurudev Dutt, J. M. Taylor, A. S. Zibrov, F. Jelezko, J. Wrachtrup, P. R. Hemmer, and M. D. Lukin, Science 314, 281 (2006)

[2] M. V. G. Dutt, L. Childress, L. Jiang, E. Togan, J. Maze, F. Jelezko, A. S. Zibrov, P. R. Hemmer, and M. D. Lukin, Science 316, 1312 (2007)

[3] T. Staudacher, F. Shi, S. Pezzagna, J. Meijer, J. Du, C. A. Meriles, F. Reinhard, and J. Wrachtrup, Science 339, 561 (2013)

[4] G. Waldherr, Y. Wang, S. Zaiser, M. Jamali, T. SchulteHerbrüggen, H. Abe, T. Ohshima, J. Isoya, J. Du, P. Neumann, et al., Nature 506, 204 (2014)

[5] M. W. Doherty, N. B. Manson, P. Delaney, F. Jelezko, J. Wrachtrup, and L. C. Hollenberg, Physics Reports 528, 1 (2013)

[6] L. Childress and R. Hanson, MRS Bulletin 38, 134 (2013)
[7] S. Hong, M. S. Grinolds, L. M. Pham, D. L. Sage, L. Luan, R. L. Walsworth, and A. Yacoby, MRS Bulletin 38, 155 (2013).

[8] R. Schirhagl, K. Chang, M. Loretz, and C. L. Degen, Annual Review of Physical Chemistry 65, 83 (2014)

[9] P. Neumann, N. Mizuochi, F. Rempp, P. Hemmer, H. Watanabe, S. Yamasaki, V. Jacques, T. Gaebel, F. Jelezko, and J. Wrachtrup, Science 320, 1326 (2008).

[10] T. H. Taminiau, J. Cramer, T. van der Sar, V. V. Dobrovitski, and R. Hanson, Nature nanotechnology 9, 171 (2014)

[11] J. Zhang and D. Suter, Phys. Rev. Lett. 115, 110502 (2015)

[12] G. D. Fuchs, G. Burkard, P. V. Klimov, and D. D. Awschalom, Nature Physics 7, 789 (2011).

[13] P. C. Maurer, G. Kucsko, C. Latta, L. Jiang, N. Y. Yao, S. D. Bennett, F. Pastawski, D. Hunger, N. Chisholm, 
M. Markham, D. J. Twitchen, J. I. Cirac, and M. D. Lukin, Science 336, 1283 (2012)

[14] J. H. Shim, I. Niemeyer, J. Zhang, and D. Suter, Phys. Rev. A 87, 012301 (2013).

[15] R. Fischer, C. O. Bretschneider, P. London, D. Budker, D. Gershoni, and L. Frydman, Phys. Rev. Lett. 111, 057601 (2013)

[16] G. A. Álvarez, C. O. Bretschneider, R. Fischer, P. London, H. Kanda, S. Onoda, J. Isoya, D. Gershoni, and L. Frydman, Nature communications 6, 8456 (2015).

[17] D. Pagliero, K. R. K. Rao, P. R. Zangara, S. Dhomkar, H. H. Wong, A. Abril, N. Aslam, A. Parker, J. King, C. E. Avalos, A. Ajoy, J. Wrachtrup, A. Pines, and C. A. Meriles, Phys. Rev. B 97, 024422 (2018).

[18] A. Ajoy, R. Nazaryan, K. Liu, X. Lv, B. Safvati, G. Wang, E. Druga, J. A. Reimer, D. Suter, C. Ramanathan, C. A. Meriles, and A. Pines, Proceedings of the National Academy of Sciences 115, 10576 (2018)

[19] V. Jacques, P. Neumann, J. Beck, M. Markham, D. Twitchen, J. Meijer, F. Kaiser, G. Balasubramanian, F. Jelezko, and J. Wrachtrup, Phys. Rev. Lett. 102, 057403 (2009)

[20] P. Neumann, J. Beck, M. Steiner, F. Rempp, H. Fedder, P. R. Hemmer, J. Wrachtrup, and F. Jelezko, Science 329, $542(2010)$

[21] D. Pagliero, A. Laraoui, J. D. Henshaw, and C. A. Meriles, Applied Physics Letters 105, 242402 (2014)

[22] T. Chakraborty, J. Zhang, and D. Suter, New Journal of Physics 19, 073030 (2017).
[23] N. Xu, Y. Tian, B. Chen, J. Geng, X. He, Y. Wang, and J. Du, Phys. Rev. Applied 12, 024055 (2019).

[24] A. Dréau, J.-R. Maze, M. Lesik, J.-F. Roch, and V. Jacques, Phys. Rev. B 85, 134107 (2012).

[25] A. Dréau, P. Spinicelli, J. R. Maze, J.-F. Roch, and V. Jacques, Phys. Rev. Lett. 110, 060502 (2013)

[26] N. Aslam, G. Waldherr, P. Neumann, F. Jelezko, and J. Wrachtrup, New Journal of Physics 15, 013064 (2013).

[27] C. S. Shin, M. C. Butler, H.-J. Wang, C. E. Avalos, S. J. Seltzer, R.-B. Liu, A. Pines, and V. S. Bajaj, Phys. Rev. B 89, 205202 (2014)

[28] X.-F. He, N. B. Manson, and P. T. H. Fisk, Phys. Rev. B 47, 8816 (1993)

[29] S. Felton, A. M. Edmonds, M. E. Newton, P. M. Martineau, D. Fisher, D. J. Twitchen, and J. M. Baker, Phys. Rev. B 79, 075203 (2009).

[30] M. Chen, M. Hirose, and P. Cappellaro, Phys. Rev. B 92, 020101(R) (2015)

[31] J. H. Shim, B. Nowak, I. Niemeyer, J. Zhang, F. D. Brandao, and D. Suter, arXiv:1307.0257 [quant-ph] (2013).

[32] K. R. K. Rao and D. Suter, Phys. Rev. B 94, 060101(R) (2016)

[33] S. Simmons, R. M. Brown, H. Riemann, N. V. Abrosimov, P. Becker, H.-J. Pohl, M. L. W. Thewalt, K. M. Itoh, and J. J. L. Morton, Nature 470, 69 (2011).

[34] G. Waldherr, P. Neumann, S. F. Huelga, F. Jelezko, and J. Wrachtrup, Phys. Rev. Lett. 107, 090401 (2011) 